\title{
Rol del maestro en la inclusión educativa de los alumnos con trastornos de la comunicación oral
}

\section{Teacher's Role in the Educational Inclusion of Students with Oral Communication Disorders}

\author{
Merling Murguia* \\ Universidad Central "Marta Abreu" de Las Villas, Villa Clara, Cuba \\ ORCID: https://orcid.org/0000-0003-4705-2329 \\ Tania Hernández \\ Universidad Central "Marta Abreu" de Las Villas, Villa Clara, Cuba \\ ORCID: https://orcid.org/0000-0002-0409-9269 \\ Tamara B. Hernández \\ Universidad Central "Marta Abreu" de Las Villas, Villa Clara, Cuba \\ ORCID: https://orcid.org/0000-0002-2643-673X \\ Mercedes A. Carrera \\ Universidad Central "Marta Abreu" de Las Villas, Villa Clara, Cuba \\ ORCID: https://orcid.org/0000-0002-5945-6577
}

Received 06-12-20 Revised 07-25-20 Accepted 09-12-20 On line 09-30-20

*Correspondence

Email: mmurgia@uclv.cu
Cite as:

(C) Universidad San Ignacio de Loyola, Vicerrectorado de Investigación, 2020 


\section{Resumen}

La asunción de nuevas interpretaciones con respecto al término inclusión educativa y su evolución dentro de los contextos educativo y social, impone cambios a la visión y la labor del maestro. Numerosos investigadores de diversas latitudes han escrito acerca de la definición de inclusión educativa, sus características, las barreras que impiden su concreción, las experiencias positivas y buenas prácticas existentes; realizando aportes que se convierten en fundamentos teóricometodológicos a tomar en consideración. Sin embargo, se ha podido constatar que no existen suficientes propuestas científicas referidas al redimensionamiento del rol del maestro para favorecer la inclusión educativa de los alumnos con trastornos de la comunicación oral. El objetivo de este artículo, basado en una investigación predominantemente cualitativa, con la investigación acción colaborativa como estrategia metodológica, y a partir de la aplicación de una encuesta tipo Likert, es diagnosticar las actitudes de los maestros de educación primaria con respecto a la inclusión educativa de los alumnos con trastornos de la comunicación oral. Sobre la base de los resultados obtenidos se ofrecen criterios para el redimensionamiento del rol del maestro en función de este propósito. El estudio que se presenta podría enriquecer y transformar aspectos significativos en la aspiración de construir escuelas cada vez más inclusivas.

Palabra clave: Inclusión educativa; Rol del maestro; Trastornos de la comunicación oral.

\section{Summary}

The assumption of new interpretations regarding the term educational inclusion and its evolution within the educational and social contexts imposes changes to the vision and work of the teacher. Numerous researchers from different latitudes have written about the definition of educational inclusion, its characteristics, the barriers that impede its concretion, the positive experiences and good existing practices; making contributions become theoretical-methodological foundations to take into account. However, it has been found that there are not enough scientific proposals regarding the resizing of the role of the teacher to favor the educational inclusion of students with oral communication disorders. The objective of this article, based on predominantly qualitative research, with collaborative action research as a methodological strategy, and from the application of a Likert-type survey, is to diagnose the attitudes of primary school teachers regarding the educational inclusion of students with oral communication disorders. On the basis of the results obtained, criteria for resizing the role of the teacher are offered based on this purpose. The study presented could enrich and transform significant aspects in the aspiration to build more and more inclusive schools.

Keywords: Educational Inclusion; Teacher's role; Oral Communication Disorders.

\section{Introducción}

La inclusión educativa ha alcanzado una connotación especial en el contexto educativo de los países del mundo y especialmente en el cubano, transformando los conocimientos, percepciones, modos de actuación y prácticas cotidianas de sus co-protagonistas.

Constituye un proceso (Ainscow, 2004; Escribano y Martínez, 2013; Reyes 2017) que reclama la unidad en su comprensión y la toma de decisiones por parte de cada uno de los participantes.

Su necesidad ha sido avalada legalmente a nivel internacional en diferentes espacios y documentos: la Conferencia Mundial sobre Educación para todos (Organización de las Naciones 
Unidas para la Educación, la Ciencia y la Cultura [UNESCO], 1990), la Declaración de Salamanca (UNESCO, 1994), el Marco de Acción de Dakar (UNESCO, 2000), entre otros.

Por ello, a partir de estos eventos y declaraciones, se estableció el compromiso de asegurar una educación básica de calidad a niños, niñas, jóvenes y adultos. (Viera y Zeballos, 2014).

El término inclusión, considerado inicialmente como integración educativa, surge en la década de los 60, ante la búsqueda de un espacio o colocación en la enseñanza regular para los alumnos que tradicionalmente asistían a la enseñanza especial (De la Oliva, Tobón, Pérez y Romero, 2015).

Esta posición, en su momento, revolucionó los sistemas educativos ya que defendía los derechos de los alumnos con discapacidad a asistir a instituciones de la educación general, poniendo el punto de mira en la solución a las dificultades en la enseñanza como vía para eliminar las barreras para el aprendizaje y la participación.

Tres décadas después, aproximadamente, comienza el aclamo por la inclusión, como concepto "...más amplio que el de integración y parte de un supuesto distinto, porque está relacionada con la naturaleza misma de la educación general y de la escuela común" (Blanco, 1999, p.62). Se comprende, además, como un proceso que reclama educación para todos, asumida como un derecho de todos y no como el privilegio de pocos, que exige el acceso a una educación básica para todas las personas. (Muñoz, 2003 y Arnaiz, 2004).

Desde esta perspectiva, se asume la inclusión educativa como un proceso dirigido a la eliminación de barreras, la garantía de la permanencia y el logro del éxito de todos los alumnos sin importar el tipo de escuela o enseñanza. Por lo que se defiende su relación directa con la participación, en aras de que los alumnos, en las escuelas regulares, participen y aprendan en igualdad de condiciones como evidencia de su inclusión social, considerada como otra de las visiones de dicho término.

De esta manera la inclusión educativa no está centrada en el tipo de escuela; especial o regular, sino en el alcance de objetivos socioeducativos por todos los alumnos independientemente de la modalidad o institución escolar que brinda la oferta educativa (Borges y Orosco, 2014, p.14).

Estos criterios reafirman la necesidad de poner la mira en los alumnos que presentan trastornos de la comunicación oral, por la incidencia que estos pueden tener en el cumplimiento, con calidad, de los objetivos socioeducativos delimitados para cada nivel educativo.

Los cambios que han ocurrido y que deben ocurrir en la manera de entender y atender a los alumnos con trastornos de la comunicación oral están directamente relacionados con el incremento y la diversidad de estos que asisten a las escuelas regulares y con la adopción de un modelo inclusivo.

Este modelo presupone tanto la construcción de un vínculo entre los maestros y cada uno de sus alumnos sobre la base del pleno reconocimiento y el respeto mutuo (López, 2016) como la necesidad de replantear muchas de las prácticas que se llevan a cabo diariamente en los contextos educativos.

En las prácticas educativas constituye una tendencia el hecho de responsabilizar a "un especialista", el logopeda, con la atención a los alumnos con trastornos de la comunicación oral. 
Es común que, desde la organización de la atención logopédica que se les brinda, se proyecten acciones y tareas que involucren a los maestros del grupo clase al que pertenece el alumno, pero no siempre se logra el comprometimiento de los maestros con la tarea que se les asigna, con el control al cumplimiento de estas por parte de los alumnos y con la evaluación conjunta maestro - logopeda) del desarrollo alcanzado por estos últimos (Murguia, Veitía, Hernández y Carrera, 2019).

Esta actitud atenta contra la inclusión educativa de los alumnos, si se toma en cuenta que para lograrla "necesitamos maestros y profesores ágiles de cuerpo y mente [...] que den respuestas educativas adecuadas a cada niño en particular y al grupo clase" (Pernas, 2009, p.164).

Una propuesta de solución debe estar dirigida a redimensionar el rol del maestro, que no significa, necesariamente, "volver a una situación anterior donde... tenía otro lugar y su trabajo era más valorado" (Deneo, 2016, p. 86) ni "agregar" otras funciones a las que ya este tiene de modo que se le dificulte cumplir con unas y otras.

Se trata de promover acciones conjuntas que posibiliten la dirección de un proceso pedagógico individualizado, sustentado en el compromiso, la unidad de convicciones, el establecimiento de políticas que transformen las prácticas educativas y que conlleven a la existencia de maestros capaces de poner en marcha la inclusión educativa desde su labor (Oliver, 2003; Gento, 2007; Aguerrondo citado en Deneo, 2016).

En el contexto educativo cubano, esta es una demanda que requiere de una incursión en el análisis crítico del rol de los maestros para favorecer la inclusión educativa de los alumnos con trastornos de la comunicación oral, estimulada por la escasa existencia de investigaciones previas relacionadas con el tema.

Por esta razón, el estudio que se presenta, con el objetivo de diagnosticar las actitudes de los maestros de educación primaria con respecto a la inclusión educativa de los alumnos con trastornos de la comunicación oral, constituye en sí mismo un novedoso aporte que podría enriquecer y transformar aspectos significativos en la aspiración de construir escuelas cada vez más inclusivas.

\section{Método}

La investigación tiene como antecedentes la experiencia de las autoras como logopedas en instituciones de la enseñanza regular y/o especial, como miembros de diferentes proyectos investigativos relacionados con la atención a niños, adolescentes y jóvenes con necesidades educativas especiales en el lenguaje y la comunicación; así como su actual participación en calidad de investigadoras del Proyecto Asociado a Programa Nacional "La Pedagogía Especial como sustento para la inclusión socioeducativa de los escolares con necesidades educativas especiales" que se desarrolla en el Departamento Educación Especial, Facultad Educación Infantil, Universidad Central "Marta Abreu" de Las Villas.

Se corresponde con una investigación predominantemente cualitativa, con la investigación-acción-colaborativa (IAC), como estrategia metodológica, pues a partir de los criterios de Bartolomé, 1997, esta:

Se da cuando algunos miembros de dos o más instituciones (generalmente una de ellas más orientada a la producción de investigación científica o a la formación de profesorado y la otra una escuela o una institución en la que trabajan esos profesionales a los que se quiere formar) deciden agruparse para resolver juntos problemas que atañen a la práctica profesional de estos últimos, vinculando los procesos de investigación con los procesos de innovación y con el desarrollo y formación profesional. (p.385) 
En este caso, las autoras del presente trabajo, profesoras de la universidad anteriormente mencionada, y un grupo de logopedas que trabajan en diferentes escuelas, deciden agruparse para resolver juntos un problema que atañe a la práctica profesional de estos últimos, asociado a la búsqueda de propuestas que favorezcan la inclusión educativa de los alumnos de la escuela primaria con trastornos de la comunicación oral.

Este grupo de profesionales armonizan, coordinan sus conocimientos, esfuerzos y energías a fin de percibir y comprender sus experiencias, acciones, emociones, motivaciones, etcétera, cuando el equipo integrado delimita el marco de problemas motivo de estudio, construye en forma crítica y cooperativamente el proceso para efectuar la indagación, y así poder encauzar las acciones hacia la obtención de un fin u objetivo común: la construcción de nuevo conocimiento; logrando también el aprendizaje de una metodología de comunicación y acción compartida. (Cano y García, 2010).

En correspondencia, se conforma un grupo de discusión, entendido como:

...un método de investigación dialógico, basado en la producción de discursos, consistente en reunir a un grupo de seis a diez personas y suscitar entre ellas una discusión sobre el tema de interés, que debe estar dirigida por un/a moderador/a. La gran variedad de discursos resultantes de su aplicación serán la fuente de materia prima fiable para el análisis e interpretación de los resultados. (López, 2010, p.150).

Este grupo de discusión está integrado por 10 logopedas que laboran en diferentes escuelas primarias de la provincia de Villa Clara. En su selección se considera que:

- Son los que atienden directamente a los alumnos con trastornos de la comunicación oral.

- Entre sus funciones se encuentra la orientación a otros maestros, directivos, familias.

- Y, por la naturaleza de su trabajo, tienen una percepción más real con respecto a las limitaciones que aún existen en el rol de los maestros para favorecer la inclusión educativa de los alumnos con trastornos de la comunicación oral.

Los miembros de este grupo de discusión, además, son graduados que poseen conocimientos generales y actualizados sobre la inclusión educativa, recibidos en su formación inicial; en su etapa estudiantil formaron parte de grupos de investigación científico estudiantil vinculados a este tema y sobre el mismo realizaron sus tesis de culminación de estudios.

Considerando estos criterios, después de realizados tres talleres como parte de un curso de postgrado titulado "La inclusión educativa de los alumnos con trastornos de la comunicación oral", se convoca al grupo de discusión con el propósito de elaborar una encuesta, para el diagnóstico de las actitudes de los maestros de educación primaria con respecto a la inclusión educativa de los alumnos con trastornos de la comunicación oral.

En relación con el tema de inclusión educativa, la actitud es entendida como: “...un conjunto de percepciones, creencias, sentimientos a favor o en contra y formas de reaccionar ante la postura educativa que centra su esfuerzo en el logro de los aprendizajes de todos los estudiantes" (Granada, Pomés y Sanhueza, 2013, p.53).

La encuesta tipo Likert se asume como un instrumento psicométrico donde el encuestado debe indicar su acuerdo o desacuerdo sobre cada ítem, a partir de una escala ordenada y unidimensional (Bertram, 2008). Según Cañadas y Sánchez-Bruno (1998) y Dawes (1975) 
constituye uno de los instrumentos que suele ser reconocido entre los más utilizados para la medición en Ciencias Sociales.

Su elaboración por parte del grupo de discusión tiene como antecedentes la revisión y análisis crítico de publicaciones e investigaciones nacionales e internacionales. Se parte del análisis colectivo de los indicadores de las tres dimensiones que establece el IndexforInclusion: crear culturas inclusivas, elaborar políticas inclusivas y desarrollar prácticas inclusivas (Booth y Ainscow, 2002, 2011; Booth, Ainscow y Kingston, 2006), asumidas en la investigación como categorías.

Las autoras del presente artículo, convertidas en moderadoras, propician la participación de los integrantes del grupo para que expresen libremente su opinión respecto a qué ítems considerar como imprescindibles para la elaboración de la encuesta tipo Likert.

Todos los criterios quedan registrados para su posterior valoración. En el análisis se estudia tanto los temas surgidos y contenidos expresados, como la forma de expresarse, su momento y situación creada, las respuestas a las opiniones de los demás, donde todos los miembros están de acuerdo con las conclusiones o juicios emitidos.

De esta forma, los indicadores presentados inicialmente son enriquecidos y se determina para la encuesta tipo Likert un total de 19 ítems. De ellos siete se corresponden con la dimensión crear culturas inclusivas, seis con la de elaborar políticas inclusivas y otros seis con desarrollar prácticas inclusivas. Se ofrecen las opciones totalmente de acuerdo (TA), de acuerdo (A), indiferente (I), desacuerdo (D) y totalmente en desacuerdo (TD).

Tabla 1.

Encuesta a aplicada a los profesionales

\begin{tabular}{|c|c|c|c|c|c|c|}
\hline Categorías & & $\begin{array}{l}\text { totalmente } \\
\text { de acuerdo } \\
\text { (TA) }\end{array}$ & $\begin{array}{l}\text { de } \\
\text { acuerdo } \\
\text { (A) }\end{array}$ & $\begin{array}{l}\text { indiferente } \\
\text { (I) }\end{array}$ & $\begin{array}{l}\text { desacuerdo } \\
\text { (D) }\end{array}$ & $\begin{array}{l}\text { totalmente } \\
\text { en } \\
\text { desacuerdo } \\
\text { (TD) }\end{array}$ \\
\hline \multirow{4}{*}{ 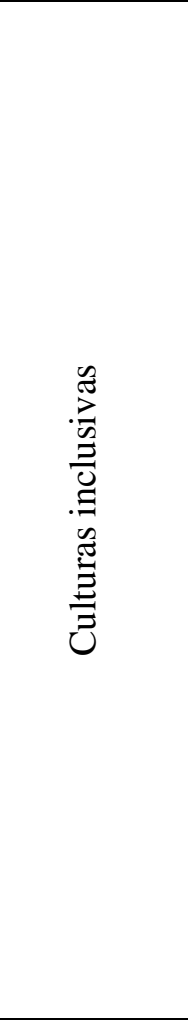 } & $\begin{array}{l}\text { 1. La inclusión } \\
\text { educativa es una } \\
\text { tendencia pedagógica } \\
\text { asumida en la atención } \\
\text { a los alumnos con } \\
\text { trastornos de la } \\
\text { comunicación oral. }\end{array}$ & & & & & \\
\hline & $\begin{array}{l}\text { 2. Los alumnos con } \\
\text { trastornos de la } \\
\text { comunicación oral } \\
\text { tienen que asistir a } \\
\text { escuelas del lenguaje. }\end{array}$ & & & & & \\
\hline & $\begin{array}{l}\text { 3. La atención a los } \\
\text { alumnos con trastornos } \\
\text { de la comunicación } \\
\text { oral le corresponde al } \\
\text { logopeda. }\end{array}$ & & & & & \\
\hline & $\begin{array}{lr}\text { 4. Los docentes deben } \\
\text { conocer } & \text { las } \\
\text { características de } & \text { los } \\
\text { escolares } & \text { con } \\
\text { trastornos de } & \text { la } \\
\text { comunicación oral. }\end{array}$ & & & & & \\
\hline
\end{tabular}




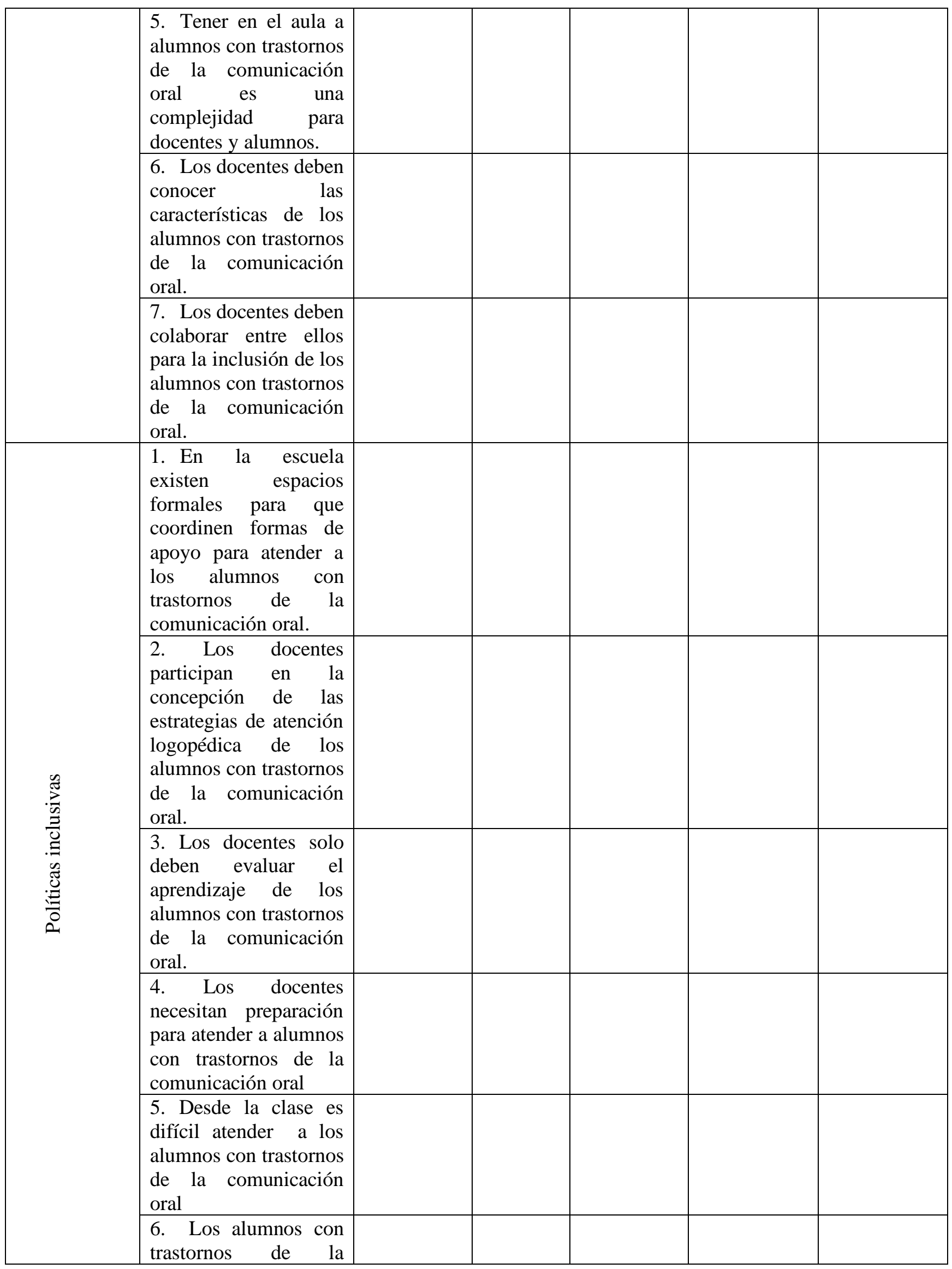




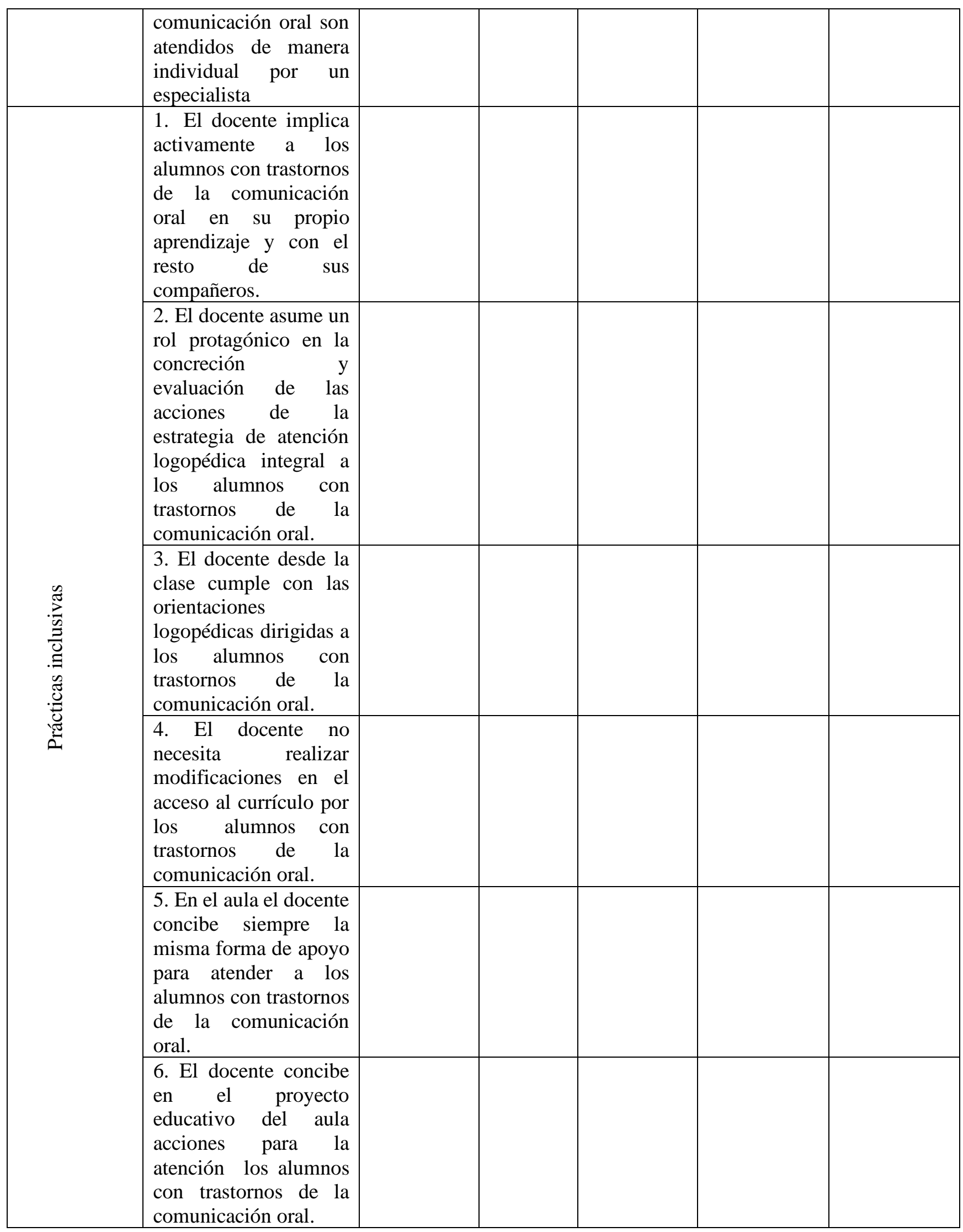

Fuente: elaboración propia

Posteriormente, se emplea el grupo focal considerado como un método de investigación colectivista, más que individualista, que se centra en la pluralidad y variedad de las actitudes, 
experiencias y creencias de los participantes, en un espacio de tiempo relativamente corto. (Martínez, 2000).

El grupo focal está conformado por uno de los logopedas del grupo de discusión antes mencionado, un psicopedagogo, cinco maestros y tres directivos pertenecientes al mismo centro donde labora el primero.

Entre los integrantes del grupo focal, si bien todos son maestros, existen diferencias respecto a los años de experiencia profesional, por lo que necesariamente no han recibido en su formación contenidos relacionados con la inclusión educativa.

Poseen diferentes perfiles profesionales (una profesora de computación, siete maestras de diferentes grados, una psicopedagoga y un logopeda); y, aunque interactúan cotidianamente con alumnos que poseen trastornos de la comunicación oral, la diversidad de las funciones que les corresponde desempeñar, les permite poseer criterios disímiles acerca del rol de los coprotagonistas del proceso; lo que incide en la percepción que tienen con respecto a la inclusión educativa.

Para comparar y contrastar la información obtenida desde distintos ángulos, se utiliza la triangulación de fuentes de datos o recogida de información de diversas procedencias (Báxter, 2003), en este caso de los integrantes del grupo focal.

El análisis de los datos aportados por las diferentes fuentes se realiza mediante la consideración de las selecciones realizadas por los encuestados, en correspondencia con las opciones definidas en cada uno de los ítems de las categorías (culturas inclusivas, políticas inclusivas y prácticas inclusivas):

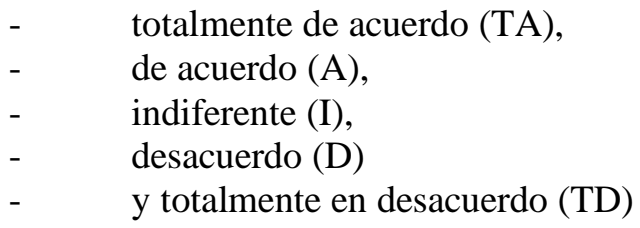

Así, el empleo de la encuesta tipo Likert posibilitó tener un conocimiento más profundo respecto a las actitudes que muestran los diferentes maestros hacia la inclusión educativa de los alumnos con trastornos de la comunicación oral.

\section{Resultados}

Para sintetizar la información se ofrece la valoración obtenida con respecto a los ítems que revelan el camino a seguir para lograr la transformación de la actitud de los maestros frente a la inclusión educativa, en general, y particularmente la de los alumnos con trastornos de la comunicación oral.

Con respecto a la categoría culturas inclusivas los ítems seleccionados con criterios desfavorables se corresponden con la comprensión de la inclusión como un proceso (3D y 1I), la responsabilidad absoluta del logopeda en la atención a los alumnos con trastornos de la comunicación oral (2TA y 2A ), la complejidad que se le atribuye a la labor del maestro en la atención de dichos alumnos en el contexto áulico (2TA y 2A) y la importancia de la colaboración entre todos los maestros para la inclusión de los mismos (4D y 1I).

Al valorar los resultados obtenidos en la categoría políticas inclusivas se muestra un consenso (10TD) con respecto a la existencia de espacios formales en los que se coordinen acciones para atender a los alumnos con trastornos de la comunicación oral y la participación de 
los maestros en la concepción de las estrategias de atención logopédica a estos. En cuanto al ítem referido a la atención individual y especializada que deben recibir estos alumnos fue votado con 2A y 1TA. El resto de los ítems fueron marcados favorablemente.

Los resultados alcanzados en la categoría prácticas inclusivas demuestran que existe un mayor avance en la concreción de acciones en aras de la inclusión, lo que se contradice con las carencias ya declaradas en las categorías anteriores. Solo reciben votaciones desfavorables los ítems: rol protagónico del maestro en la concreción y evaluación de las acciones para la inclusión educativa de los alumnos con trastornos de la comunicación oral (3D) y la necesidad de la implementación de estrategias para el acceso al currículo y el alcance del éxito por parte de estos alumnos (2D y $1 \mathrm{TD})$.

El análisis expuesto dirige la discusión a los siguientes puntos clave:

- Comprensión de la inclusión como un proceso.

- Rol del maestro en la inclusión educativa de los alumnos con trastornos de la comunicación oral.

\section{Discusión}

La concepción que sobre la inclusión educativa posee el maestro determina, en gran medida, el nivel de compromiso, apoyo y entrega para alcanzar los cambios que este impone.

Encontrarse con visiones reduccionista es común, si se considera que las mismas están estrechamente ligadas a la formación inicial: el maestro que en su formación recibe contenidos relacionados con la inclusión educativa tiene un espectro más amplio de lo que implica atender a la diversidad.

Al respecto, se destacan los estudios de Granada, Pomés y Sanhueza (2013) que reafirman la importancia de que los maestros posean tanto una formación inicial como continua que les permita contar con herramientas para dar respuestas educativas de calidad a la diversidad de alumnos en el proceso de enseñanza-aprendizaje y, por otra parte, les permita capacitarse y actualizarse permanentemente, para atender distintas demandas emergentes.

Se reconoce, en este sentido, que los más necesitados de esta formación, según los planes de estudios anteriores, son los maestros de educación primaria. (Arnaiz, 2003; Jiménez y Cardona citados en Sanchez, Díaz, Sanhueza \& Friz, 2008).

Los resultados obtenidos, denotan falta de conocimiento sobre la evolución del concepto inclusión, visto aún como la integración de personas con discapacidad; lo que impide una adecuada percepción del mismo desde el principio educación para todos internacionalmente avalado por UNESCO, 1990, 1994 y 2000.

Un primer paso para el cambio es lograr una cultura inclusiva, lo que conlleva a transformar y actualizar los conocimientos que tiene el maestro sobre la inclusión educativa; entendiéndola como un proceso:

orientado a responder a la diversidad del alumnado, incrementando su participación y reduciendo la exclusión en y desde la educación, cuya principal finalidad es garantizar una educación de calidad a todos los estudiantes, asegurando su presencia, participación y aprendizaje, con especial énfasis en aquellos que, por diferentes razones, están excluidos o en riesgo de ser excluidos/marginados (Booth\& Ainscow, 2004; UNESCO, 2005). 
dirigido a dar respuesta a la diversidad de necesidades de todos los alumnos. (Escribano \& Martínez, 2013)

que promueve "la presencia, la participación y el rendimiento de todos los estudiantes de su localidad, incluidos aquellos más vulnerables a los procesos de exclusión, aprendiendo de esa forma a vivir con la diferencia" (Reyes, 2017, p. 6).

En correspondencia, el maestro de la escuela primaria debe comprender la inclusión educativa como un proceso que promueve el aprendizaje y la participación de todos los alumnos en riesgo de exclusión, y no solamente se refiere a aquellos alumnos con necesidades educativas especiales asociadas a discapacidad.

No basta solo con lograr esta percepción, se requiere que el maestro posea conocimientos de cada uno de sus alumnos, sus intereses, motivaciones, necesidades y potencialidades, para organizar estrategias de enseñanza que permitan brindarles las oportunidades y los apoyos necesarios para alcanzar los objetivos (Borges \& Orosco, 2014). Este criterio contradice los manifestados por los maestros con respecto a la absoluta responsabilidad del logopeda en la inclusión educativa de los alumnos con trastornos de la comunicación oral.

Existen propuestas como la de España \& Castejón (2004), que apoyan la colaboración del logopeda con el maestro y promueven la utilización del espacio áulico para ejercer la influencia educativa. Un estudio previo realizado por parte de las autoras reconoce y ejemplifica cómo el trabajo colaborativo entre maestros, directivos, especialistas y logopeda es posible, considerando el rol de cada uno y complementando sus conocimientos, experiencias y prácticas cotidianas. (Murguia, Veitía, Hernández \& Carrera, 2019).

Otro ejemplo lo ofrece Acosta (2006) al definir los diferentes roles que pueden asumir los maestros, los logopedas, los psicopedagogos en colaboración. A juicio de las autoras, estos pudieran favorecer la inclusión educativa de los alumnos con trastornos de la comunicación oral, con la realización de determinadas adecuaciones ajustadas al contexto de la investigación. Se ilustra en la siguiente tabla:

\section{Tabla 2.}

Rol de los profesionales en la inclusión educativa de los alumnos con trastornos de la comunicación oral.

\begin{tabular}{|c|c|c|c|}
\hline Logopeda & Directivos & Maestros & Psicopedagogo \\
\hline $\begin{array}{l}\text { Ofrece información } \\
\text { sobre la adquisición y } \\
\text { desarrollo del lenguaje y } \\
\text { la comunicación. }\end{array}$ & $\begin{array}{l}\text { Favorece los procesos } \\
\text { comunicativos en el } \\
\text { centro escolar. }\end{array}$ & $\begin{array}{l}\text { Ofrece información } \\
\text { sobre el diseño y } \\
\text { desarrollo del currículo } \\
\text { común. }\end{array}$ & $\begin{array}{lrr}\text { Ofrece información } \\
\text { sobre el impacto de las } \\
\text { dificultades } & & \text { del } \\
\text { lenguaje } & \text { y } & \text { la } \\
\text { comunicación } & \text { en } & \text { el } \\
\text { aprendizaje } & \text { y } & \text { el } \\
\text { desarrollo } & \text { de } & \text { la } \\
\text { personalidad } & \text { de } & \text { los } \\
\text { alumnos. } & & \\
\end{array}$ \\
\hline \begin{tabular}{lr} 
Facilita & \multicolumn{2}{r}{ información } \\
sobre las características \\
de los alumnos con \\
trastornos de la \\
comunicación oral
\end{tabular} & $\begin{array}{l}\text { Actualiza el proyecto } \\
\text { educativo del centro } \\
\text { escolar } \\
\text { correspondencia con la } \\
\text { información que recibe }\end{array}$ & $\begin{array}{l}\text { Facilita información } \\
\text { sobre las actividades y } \\
\text { tareas docentes de la } \\
\text { clase, }\end{array}$ & $\begin{array}{l}\text { Facilita información } \\
\text { sobre la vulnerabilidad } \\
\text { de los alumnos con } \\
\text { trastornos de la } \\
\text { comunicación oral en } \\
\text { las relaciones con el }\end{array}$ \\
\hline
\end{tabular}




\begin{tabular}{|c|c|c|c|}
\hline & $\begin{array}{l}\text { de los diferentes } \\
\text { profesionales. }\end{array}$ & & $\begin{array}{l}\text { grupo-clase y el acceso } \\
\text { al contenido. }\end{array}$ \\
\hline $\begin{array}{l}\text { Ordena, dirige y realiza } \\
\text { la investigación } \\
\text { logopédica }\end{array}$ & $\begin{array}{lr}\text { Favorece } & \text { la } \\
\text { organización escolar } \\
\text { necesaria para que se } \\
\text { cumpla con la } \\
\text { investigación } \\
\text { logopédica. }\end{array}$ & $\begin{array}{l}\text { Contribuye, enriquece } \\
\text { y participa en la } \\
\text { evaluación de algunos } \\
\text { aspectos de la } \\
\text { investigación } \\
\text { logopédica }\end{array}$ & $\begin{array}{l}\text { Participa en la } \\
\text { investigación } \\
\text { logopédica en aspectos } \\
\text { como la exploración de } \\
\text { la lectura y escritura. } \\
\text { y la valoración de } \\
\text { diferentes aspectos de } \\
\text { la personalidad. }\end{array}$ \\
\hline $\begin{array}{l}\text { Diseña las tareas y } \\
\text { acciones de la estrategia } \\
\text { de atención logopédica } \\
\text { integral }\end{array}$ & $\begin{array}{l}\text { Coordina los espacios } \\
\text { de intercambio } \\
\text { profesional y elabora } \\
\text { estrategias de } \\
\text { colaborativo. }\end{array}$ & $\begin{array}{l}\text { Participa en la } \\
\text { concepción de las } \\
\text { tareas y acciones de la } \\
\text { estrategia de atención } \\
\text { logopédica integral } \\
\text { referidas al tratamiento } \\
\text { al contenido o la } \\
\text { dirección del proceso } \\
\text { de enseñanza } \\
\text { aprendizaje. }\end{array}$ & $\begin{array}{llr}\text { Sugiere } & & \text { las } \\
\text { modificaciones } & \\
\text { necesarias y posibles } \\
\text { variantes para } \\
\text { tratamiento } \\
\text { contenido o en la } \\
\text { dirección del proceso } \\
\text { de } & & \text { al } \\
\text { aprendizaje } & & \end{array}$ \\
\hline $\begin{array}{l}\text { Brinda atención } \\
\text { logopédica directa o } \\
\text { indirecta, individual o } \\
\text { colectiva a los alumnos } \\
\text { con trastornos de la } \\
\text { comunicación oral. }\end{array}$ & $\begin{array}{l}\text { Estructura desde la } \\
\text { organización escolar la } \\
\text { atención a los alumnos } \\
\text { con trastornos de la } \\
\text { comunicación oral }\end{array}$ & $\begin{array}{l}\text { Apoya la atención } \\
\text { logopédica directa o } \\
\text { indirecta e individual o } \\
\text { colectiva con el } \\
\text { cumplimiento } \\
\text { acciones de dentro del } \\
\text { proceso de enseñanza } \\
\text { aprendizaje. }\end{array}$ & \begin{tabular}{lrr} 
Apoya la atención \\
logopédica directa & o \\
indirecta e individual o & \\
colectiva & \multicolumn{2}{r}{ con } \\
realización de & tareas \\
relacionadas con & el \\
desarrollo & de & la \\
personalidad & y & el \\
aprendizaje de & los \\
alumnos con trastornos \\
de la comunicación oral
\end{tabular} \\
\hline $\begin{array}{l}\text { Realiza su clase } \\
\text { logopédica en el espacio } \\
\text { áulico, conjuntamente } \\
\text { con el maestro }\end{array}$ & $\begin{array}{l}\text { Planifica y/o controla } \\
\text { las interacciones entre } \\
\text { los profesionales para } \\
\text { la dirección del proceso } \\
\text { der enseñanza } \\
\text { aprendizaje. }\end{array}$ & $\begin{array}{l}\text { Facilita la relación } \\
\text { entre los objetivos de la } \\
\text { clase logopédica con } \\
\text { los objetivos de la clase } \\
\text { ordinaria }\end{array}$ & $\begin{array}{l}\text { Sugiere formas de } \\
\text { trabajo individual y } \\
\text { colectivo atendiendo a } \\
\text { las características del } \\
\text { grupo-clase y de los } \\
\text { alumnos con trastornos } \\
\text { de la comunicación }\end{array}$ \\
\hline $\begin{array}{l}\text { Orienta a familias, } \\
\text { maestros y otros agentes } \\
\text { educativos }\end{array}$ & $\begin{array}{l}\text { Planifica los espacios } \\
\text { de orientación a las } \\
\text { familias, maestros y } \\
\text { otros agentes } \\
\text { educativos. }\end{array}$ & $\begin{array}{lrr}\text { Cumple } & \text { con } & \text { las } \\
\text { orientaciones } & \text { recibidas } \\
\text { por parte del logopeda. } \\
\text { Coopera } & \text { con } & \text { la } \\
\text { orientación } & \text { a } & \text { las } \\
\text { familias. } & & \\
\end{array}$ & $\begin{array}{lrr}\text { Coopera } & \text { con } & \text { la } \\
\text { orientación } & \text { a } & \text { las } \\
\text { familias. } & & \end{array}$ \\
\hline $\begin{array}{l}\text { Evalúa el progreso de } \\
\text { los alumnos con } \\
\text { trastornos de la } \\
\text { comunicación oral y la } \\
\text { efectividad de la } \\
\text { estrategia de atención } \\
\text { logopédica integral }\end{array}$ & $\begin{array}{l}\text { Brinda seguimiento a la } \\
\text { evaluación de los } \\
\text { alumnos con trastornos } \\
\text { de la comunicación oral } \\
\text { y actualiza las } \\
\text { estrategias de trabajo } \\
\text { colaborativo. }\end{array}$ & $\begin{array}{l}\text { Evalúa el progreso de } \\
\text { los alumnos con } \\
\text { trastornos de la } \\
\text { comunicación oral y la } \\
\text { efectividad de la } \\
\text { estrategia de atención } \\
\text { logopédica integral }\end{array}$ & $\begin{array}{l}\text { Evalúa el progreso de } \\
\text { los alumnos con } \\
\text { trastornos de la } \\
\text { comunicación oral y la } \\
\text { efectividad de la } \\
\text { estrategia de atención } \\
\text { logopédica integral }\end{array}$ \\
\hline $\begin{array}{l}\text { Fomenta el trabajo } \\
\text { colaborativo con otros } \\
\text { docentes y las familias }\end{array}$ & $\begin{array}{l}\text { Fomenta el trabajo } \\
\text { colaborativo con otros } \\
\text { docentes y las familias }\end{array}$ & $\begin{array}{l}\text { Fomenta el trabajo } \\
\text { colaborativo con otros } \\
\text { docentes y las }\end{array}$ & $\begin{array}{l}\text { Fomenta el trabajo } \\
\text { colaborativo con otros } \\
\text { docentes y las }\end{array}$ \\
\hline
\end{tabular}


familias

familias

Fuente: elaboración propia, a partir de los criterios de Acosta (2006)

Resalta entonces como prioridad el establecimiento de vínculos sistemáticos y bien planificados entre los maestros y el resto de los agentes educativos para elaborar políticas inclusivas. En la encuesta aplicada este constituye el ítem más desfavorablemente marcado.

Y esa movilización adecuadamente articulada nos ha de llevar a entender que no podemos trabajar ni dar respuesta a las muchas interrogantes que la educación inclusiva va a requerir si no nos movemos todos en una misma dirección, colaborando estrechamente. (Navarro y Espino, 2012, p. $80)$.

La creación de diferentes espacios formales que les permitan a los maestros y al resto de los profesionales, incluidos los directivos, coordinar, concebir, planificar y distribuir responsabilidades y acciones con respecto a la inclusión educativa de los alumnos con trastornos de la comunicación oral, es una prioridad que debe convertirse en una dinámica de trabajo.

A decir de Acosta (2006) la colaboración educativa defiende el cambio del modelo organizativo tradicional, que en lugar de potenciar el uso de aulas especiales y el tratamiento del niño con necesidades educativas especiales separado de sus compañeros, propone una nueva situación sobre la base de tres pilares: traslado del apoyo al aula ordinaria, el diseño, desarrollo y evaluación de la enseñanza de manera colaborativa entre todos los profesionales y la atención individualizada al alumno durante períodos cortos de tiempo.

Si bien las categorías antes expuestas, culturas y políticas inclusivas, despiertan cierta polémica; las prácticas inclusivas son más problematizadoras cuando se trata de exponer o definir qué rol le corresponde al maestro en la inclusión de los alumnos con trastornos de la comunicación oral, teniendo en cuenta el cumplimiento de sus funciones y la toma de decisiones con respecto al currículo como garantía del éxito de estos alumnos.

Un punto clave es el conocimiento que debe tener el maestro de sus alumnos para poder: proporcionar una educación personalizada reconociendo que lo individual en las necesidades educativas de los alumnos, es lo referido a las posibilidades, intereses, niveles, ritmos y estilos de aprendizaje, satisfacer las necesidades individuales y especiales de los alumnos y organizar el aula de modo que favorezca a la participación y cooperación entre los alumnos (Borges y Orosco, 2014).

Otro punto a considerar es el dominio del Fin y los objetivos del nivel de enseñanza, lo que le posibilita: lograr el equilibrio que debe existir en la respuesta educativa, que proporcione, desde una estructura curricular común a todos; realizar adecuaciones curriculares en contextos escolares para los alumnos que lo requieran y cubrir con los aprendizajes esperados que marca el programa de estudio de educación primaria (Castillo, 2016).

Es importante que el maestro aprenda a proveer instrucciones claras, reorganizar constantemente el salón, usar eficientemente los recursos, tener flexibilidad ante las necesidades de los alumnos, monitorear a los estudiantes, ser abiertos a las sugerencias y a los cambios. (Rivero, 2017).

En fin, el maestro inclusivo "debe estar dispuesto a asumir riesgos y ensayar nuevas formas de enseñanza; reflexionar sobre su propia práctica para transformarla, actualizarse permanentemente y valorar las diferencias como un elemento de enriquecimiento profesional" (Rivero, 2017, p. 117). 
El estudio realizado posibilita actualizar, con el empleo de métodos científicos, el diagnóstico que se posee acerca de las actitudes de los maestros de educación primaria con respecto a la inclusión educativa de los alumnos con trastornos de la comunicación oral; para, sobre la base de los resultados obtenidos, realizar inferencias que pueden convertirse en un modesto y novedoso aporte al enriquecimiento y transformación de aspectos significativos en la aspiración de construir escuelas cada vez más inclusivas.

Para ello es imprescindible comprender la inclusión educativa de los alumnos con trastornos de la comunicación oral como un proceso, en el que participan todos los profesionales que interactúan con estos y hasta sus coetáneos; bajo la coordinación de acciones coherentemente planificadas y con el fin de garantizar el acceso, la permanencia y el éxito de estos alumnos en todos los contextos.

\section{Conclusiones}

El estudio realizado sobre las actitudes de los maestros ante la inclusión educativa de los escolares con trastornos de la comunicación permite reconocer que aún se requiere transformar la visión que poseen estos profesionales con respecto al proceso de inclusión.

Las tendencias detectadas con la aplicación de la encuesta tipo Likert corroboran que las prácticas inclusivas son cotidianas en el accionar de los maestros, aunque no siempre se corresponden con las culturas y políticas inclusivas, siendo estas últimas las que menos se aprovechan cuando de trabajo colaborativo se trata.

La adecuada asignación y ejecución de roles, unido a la oportuna transformación del accionar tanto de maestros como de otros profesionales, agrupados en el trabajo colaborativo son la clave del éxito para lograr la necesaria inclusión educativa de los escolares con trastornos de la comunicación oral.

\section{Referencias}

Acosta, V. (2006). Investigación, evaluación y colaboración profesional ante las dificultades del lenguaje. El reto hacia los modelos inclusivos. Revista Chilena de Fonoaudiología, 7(1), 7-26.

Ainscow, M. (2004). El desarrollo de sistemas educativos inclusivos: ¿Cuáles son las palancas de cambio? Journal of Educational Change, 5(4), 1-20.

Arnaiz, P. (2003). Educación inclusiva: Una escuela para todos. Málaga: Aljibe.

Arnaiz, P. (2004). La educación inclusiva: dilemas y desafíos. Revista Educación, Desarrollo y Diversidad, 7(2), 25-40.

Bartolomé, M. (1997). Metodologiaqualitativa orientada cap al canvi a la presa de decisions. Barcelona, EDIUOC

Báxter, E. (2003). El proceso de investigación en la metodología cualitativa.El enfoque participativo y la investigación acción. En M. Martínez (Ed.), Metodología de la investigación educacional. Desafíos y polémicas actuales (pp. 98-112). La Habana: Editorial Félix Varela.

Bertram, D. (2008). Likert Scales... are the meaning of life. Topicreport.

Blanco, R. (1999). Hacia una escuela para todos y con todos. Boletín Proyecto Principal de Educación en América Latina y el Caribe, 48, 55-72.

Booth, T. \& Ainscow, M. (2002). Guía para la evaluación y mejorade la educación inclusiva. Desarrollando el aprendizaje y la participación en las escuelas. Recuperado de: http://www.cepcampgib.org/noveles/files/anexos/Index_for_inclusion.pdf 
Booth, T. \&Ainscow, M. (2004). Rutas para el desarrollo de prácticas inclusivas en los sistemas educativos. Revista de Educación (327). Recuperado de: http://www.webdocente.altascapacidades.net/Articulos/PDF/Art2/3_rutas_para_el_desarrollo_de_practicas_inclusiva s_en_los_sistemas_educativos.pdf

Booth, T., Ainscow, M. \&Kingston, D. (2006). Index para la inclusión: desarrollo del juego, el aprendizaje y la participación en educación infantil. Bristol: CSIE

Booth, T. \& Ainscow. M. (2011). Index for Inclusion: Developing learning and participation in schools ( $3^{\mathrm{a}}$ ed.). Bristol: CSIE

Borges, S. \& Orosco, M. (2014). Inclusión educativa y Educación Especial. Un horizonte singular y diverso para igualar las oportunidades de desarrollo. Educación Cubana. La Habana.

Cano, M. \& García, T. (2010). La investigación colaborativa: una experiencia en el desarrollo de un proyecto educativo. Ciencia Administrativa (1),61-67.

Cañadas, I. \& Sánchez-Bruno, A. (1998). Categorías de respuestas en escalas tipo Likert. Psicothema,10(3), 623-631. Recuperado de: http://www.psicothema.es/pdf/191.pdf

Cardona, M. C. (2006). Diversidad y educación inclusiva: enfoques metodológicos y estrategias para una enseñanza colaborativa. España: Pearson Educación.

Castillo, J. R. (2016). Docente inclusivo, aula inclusiva. Revista nacional e internacional de educación inclusiva, 9(2),264-275.

Dawes, R. M. (1975). Fundamentos y técnicas de medición de actitudes. México: Limusa.

De la Oliva, D., Tobón, S., Pérez, S., Karina, A., \& Romero, J. (2015). El Proceso de Inclusión Social desde la Socioformación: Análisis de Concepciones sobre Discapacidad y Necesidades Educativas Especiales. Paradigma, 36(2), 49-73.

Deneo, G. (2016). Perspectivas acerca de la inclusión educativa (Tesis de maestría). Universidad de la República, Montevideo, Uruguay.

Escribano, A. \& Martínez, A. (2013). Inclusión Educativa y Profesorado Inclusivo. Madrid: Narcea Ediciones.

España, Y. \& Castejón, L. (2004). La colaboración logopeda-maestro hacia un modeloinclusivo de intervención en las dificultades del lenguaje. Revista de logopedia, foniatría y audiología, 24(2), 55-66.

Gento, S. (2007). Requisitos para una inclusión de calidad en el tratamiento educativo. Bordón, 59(4), 581-595.

Granada, M., Pomés, M. \& Sanhueza, S. (2013). Actitud de los profesores hacia la inclusión educativa. Papeles de Trabajo, 25, 51-59.

López, I. (2010). El grupo de discusión como estrategia metodológica de investigación: aplicación a un caso. Edetania 38, 147-156.

López, N. (2016). Inclusión educativa y diversidad cultural en América Latina. Revista Española de Educación Comparada, (27), 35-52. Recuperado de: http://revistas.uned.es/index.php/REEC/article/view/15034/14413

Martínez, M. (2000). Los grupos focales de discusión como método de investigación. Recuperado de: http://miguelmartinezm.atspace.com/gruposfocales.html

Muñoz, C. (2003). La inclusión socio-educativa desde la práctica pedagógica formación de profesores. Revista Horizontes Educacionales, (8), 51-56.

Murguia, M., Veitía, Y., Hernández, T. \& Carrera, M. A. (2019). Escolares primarios con dislalia: una propuesta para su inclusión educativa. En Actas II del XVI Simposio de Comunicación Social: Lingüística, Medios Masivos, Arte, Etnología, Folclor y otras ciencias afines. (pp. 596-600). Santiago de Cuba: Ediciones Centro de Linguística Aplicada.

Navarro, D. \& Espino, M.A. (2012). Inclusión educativa, ¿es posible? Edetania 41, 71-81.

Oliver, M. (2003). Estrategias didácticas y organizativas ante la diversidad: dilemas del profesorado. Barcelona: Octaedro.

Pernas, C. M. (2009). La atención a la diversidad en los centros escolares. Normalización e inclusión del alumnado con necesidades específicas de apoyo educativo (ANEAE). Zona Próxima, (11), 160-169. 
Reyes, J.L. (2017). La inclusión educativa de los escolares con discapacidad intelectual en la educación primaria. Revista Dilemas Contemporáneos: Educación, Política y Valores. (3), 1-14.

Rivero, J. (2017) Las buenas prácticas en Educación Inclusiva y el rol del docente. Educ@ción en Contexto, 3(extraordinario 0). 110-120.

Sánchez, A., Díaz, C., Sanhueza, S., \& Friz, M. (2008). Percepciones y actitudes de los estudiantes de pedagogía hacia la inclusión educativa. Estudios pedagógicos (Valdivia), 34(2), 169178. https://dx.doi.org/10.4067/S0718-07052008000200010

UNESCO (2005). Guidelines for inclusión: Ensuring Access to Education for All. Recuperado de: http://disabilityrightsfund.org/wp-content/uploads/2015/11/140224e.pdf

UNESCO. (1990). Declaración Mundial sobre Educación para Todos: Satisfacción de las necesidades básicas de aprendizaje. Recuperado de: https://www.humanium.org/es/wpcontent/uploads/2013/09/1990-DeclaracionMundialEducacion.pdf

UNESCO. (1994). Declaración de Salamanca y marco de acción para las necesidades educativas especiales. Recuperado de: https://unesdoc.unesco.org/ark:/48223/pf0000098427_spa

UNESCO. (2000). Marco de Acción de Dakar. Educación para todos: cumplir nuestros compromisos comunes. Recuperado de: https://unesdoc.unesco.org/ark:/48223/pf0000121147_spa

Viera, A. \&Zeballos, Y. (2014). Inclusión educativa en Uruguay: una revisión posible. Revista Psicología, Conocimiento y Sociedad, 4(2), 237-260. 